\title{
Jueming prescription and its ingredients, semen cassiae and Rhizoma Curcumae Longae, stimulate lipolysis and enhance the phosphorylation of hormone-sensitive lipase in cultured rat white adipose tissue
}

\author{
YUE ZHANG ${ }^{1}$, JIAOJIAO LI $^{2}$ and XIUYING WEN ${ }^{3}$ \\ ${ }^{1}$ Department of Endocrinology, Liyuan Hospital, Tongji Medical College, Huazhong University of Science and \\ Technology, Wuhan, Hubei 430077; ${ }^{2}$ Department of Pediatrics, Renmin Hospital of Wuhan University, Wuhan, \\ Hubei 430060; ${ }^{3}$ Department of Traditional Chinese Medicine and Endocrinology, Liyuan Hospital, \\ Tongji Medical College, Huazhong University of Science and Technology, Wuhan, Hubei 430077, P.R. China
}

Received November 20, 2016; Accepted July 3, 2017

DOI: $10.3892 / \mathrm{mmr} .2017 .7317$

\begin{abstract}
The present study aimed to investigate the effect of jueming prescription (JMP) and its ingredients, semen cassiae (SC) and Rhizoma Curcumae Longae (RCL), on lipolysis, and to examine their effect on the phosphorylation of hormone-sensitive lipase (HSL) in cultured rat white adipose tissue (WAT). Retroperitoneal WAT was aseptically excised from adult male Sprague-Dawley rats, minced into uniform sections and subjected to ex vivo culture for $24 \mathrm{~h}$. The tissue sections were then distributed into a 24-well culture plate and treated with normal saline (vehicle), isoproterenol (ISO), JMP, SC and RCL. Non-esterified fatty acid (NEFA) and glycerol release from the intact WAT explants were determined as a measurement of lipolysis, which were measured using NEFA and glycerol assay kits. The phosphorylation of HSL at Ser563 (P-HSL S563) and 660 residues (P-HSL S660) were determined using western blot analysis. The size of the adipocytes was visualized using hematoxylin and eosin (H\&E) staining. It was found that JMP-, SC- and RCL-stimulated lipolysis was responsible for increasing the release of NEFAs and glycerol from the intact WAT in vitro. In addition, JMP, SC and RCL increased the levels of P-HSL Ser563: JMP water (JW) extract, 3.52-fold; JMP ethanol (JE) extract, 3.38-fold; SC water (SW) extract, 4.60-fold; SC ethanol (SE) extract, 4.20-fold; RCL water (RW) extract, 6.98-fold; RCL ethanol (RE) extract, 6.60-fold. JMP, SC and RCL also increased the
\end{abstract}

Correspondence to: Dr Xiuying Wen, Department of Traditional Chinese Medicine and Endocrinology, Liyuan Hospital, Tongji Medical College, Huazhong University of Science and Technology, 39 Lake Avenue, Wuhan, Hubei 430077, P.R. China

E-mail: wenxyoung@hust.edu.cn

Key words: jueming prescription, semen cassia, Rhizoma Curcumae Longae, lipolysis, hormone-sensitive lipase, white adipose tissue levels of P-HSL Ser660: JW extract, 3.16-fold; JE extract, 2.92-fold; SW extract, 4.57-fold; SE extract, 4.13-fold; RW extract, 5.41-fold; RE 4.96-fold) in the WAT. The RW extract had the most marked effect. The HE staining revealed that JMP, SC and RCL reduced the size of adipocytes in the WAT. In conclusion, JMP and its ingredients, SC and RC, stimulated lipolysis and reduced the size of adipocytes, possibly via the phosphorylation of HSL in cultured rat WAT.

\section{Introduction}

Obesity is a medical condition characterized by the expansion of adipose tissue, which is induced by the accumulation of triglycerides (TGs) in adipocytes (1). The adipocytes of white adipose tissue (WAT) are a site of fat storage, as lipid droplets and the balance of lipids stored within the lipid droplets are controlled by TG synthesis (lipogenesis) and degradation (lipolysis). Lipolysis is a biochemical process involving the breakdown of TG stored in lipid droplets, resulting in the release of non-esterified fatty acid (NEFA) and glycerol, and is one of the key processes involved in achieving weight loss (2-5). Therefore, a coordinated stimulation of lipolysis is beneficial in reducing adipose mass and preventing obesity.

Lipolysis, the catabolism of TG in WAT, is regulated by complex regulatory mechanisms. The most well known mechanism involved in mediating lipolysis is the cAMP-dependent protein kinase A (PKA) pathway (6,7). It is well-established that the PKA-mediated phosphorylation of hormone-sensitive lipase (HSL) and perilipin A are critical for stimulating lipolysis, which leads to the hydrolysis of TG and the subsequent release of NEFA and glycerol (7-12). The phosphorylation of HSL protein activates and translocates the enzyme to the surface of lipid droplets, where it hydrolyzes TG (13-17). The Ser563 and Ser660 sites of HSL are the major sites of phosphorylation associated with HSL activity $(14,18)$. HSL is phosphorylated by PKA at Ser563 and Ser660, which activates HSL, increases the translocation of HSL to lipid droplets and enhances lipolysis $(19,20)$. A previous study reported that the phosphorylation of HSL at 
Ser563 and Ser660 are reduced in the WAT of obese rats, and the basal phosphorylation of HSL at Ser563 and Ser660 is low in primary adipocytes from obese rats, compared with those from control rats (21). The phosphorylation of HSL at Ser563 and Ser660, and lipolysis are markedly increased upon isoproterenol (ISO; $\beta$-adrenergic agonist) treatment in cultured WAT explants from mice (20). ISO also promotes the phosphorylation of HSL at Ser563 and Ser660, and increases lipolysis in primary adipocytes from obese rats and non-obese rats $(21,22)$.

Chinese herbal medicines are important in the treatment of obesity. Increasing evidence from clinical studies and animal experiments study that various Chinese herbal medicines significantly promote lipolysis and the release of NEFA and glycerol in adipocytes and WAT, suppressing adipose tissue weight and body weight gain in humans and animals (2,6,23-26).

In Chinese medicine, it is believed that obesity is associated with the endogenous wet-heat and blood stasis due to the over-consumption of fatty food (27-30). Semen cassiae (SC) has the capacity to clear heat and moisten the intestines, and Rhizoma Curcumae Longae (RCL) can promote qi to activate blood and remove blood stasis $(27,28,31)$. Studies have reported that SC and RCL extracts are effective in enhancing lipolysis, and in reducing the size of adipocytes and body weight in obese rats $(2,3,6,32)$. In our previous study, the Chinese herbal medicine jueming prescription (JMP) was formulated, which consists of SC and RCL (27). Our previous studies showed that JMP reduced body weight, visceral fat weight and adipocyte size in WAT, and also alleviated liver steatosis in rats with diet-induced obesity. It was also shown to reduce rat body weights and to improve lipid metabolism in obese patients $(23,27,28)$. However, there have been no studies analyzing and comparing JMP and its ingredients, SC and RCL, on lipolysis in cultured rat WAT.

The present study investigated the effects of JMP and its ingredients, SC and RCL, on stimulating lipolysis, and whether they are responsible for increasing the release of NEFA and glycerol, and reducing the size of adipocytes and phosphorylation levels of HSL in rat WAT. This was performed using an ex-vivo WAT explant culture method.

\section{Materials and methods}

Preparation of JMP, SC and RCL. JMP is composed of SC, which is produced in Xianqiao, Zhejiang and RCL, which is produced in Qichun, Hubei, in a 1.5:1 (w/w) ratio. SC and RCL were purchased from Tianji Chinese Herbal Medicine Co., Ltd. (Wuhan, China). All herbal drugs were soaked in eight volumes of distilled water $(1: 8, \mathrm{w} / \mathrm{v})$ for $12 \mathrm{~h}$ at room temperature, followed by boiling three times ( $2 \mathrm{~h}$ each). The JMP water (JW) extract was filtered and concentrated to a concentration equivalent to $0.2 \mathrm{~g}$ raw herbs/ $1 \mathrm{ml}$ water extract (23). The JMP ethanol (JE) extract was concentrated and precipitated with $60 \%$ ethanol for $12 \mathrm{~h}$. The solution was evaporated under reduced pressure $(\sim 0.1 \mathrm{MPa})$ to produce a concentration equivalent to $0.2 \mathrm{~g}$ raw herbs in $1 \mathrm{ml}$ ethanol extract (33). The SC (SW) extract $(0.3 \mathrm{~g} / \mathrm{ml})$ and SC ethanol (SE) extract $(0.3 \mathrm{~g} / \mathrm{ml}), \mathrm{RCL}$ water $(\mathrm{RW})$ extract $(0.4 \mathrm{~g} / \mathrm{ml})$ and RCL ethanol (RE) extract $(0.4 \mathrm{~g} / \mathrm{ml})$ were prepared using the same method used for the JW extract and JE extract. The extracts were stored at $4^{\circ} \mathrm{C}$ until they were used.
Chemicals and reagents. M199 medium was from Gibco; Thermo Fisher Scientific, Inc. (Waltham, MA, USA), bovine serum albumin (BSA) and 4-(2-hydroxyethyl) piperazine-1-ethanesulfonic acid sodium salt (HEPES) were from Sigma-Aldrich; Merck Millipore (Darmstadt, Germany). The glycerol assay kit and NEFA assay kit were from Applygen Technologies Inc. (Beijing, China). ISO was from Hefeng Pharmaceutical Co., Ltd. (Shanghai, China), $100 \mu \mathrm{g} / \mathrm{ml}$ streptomycin and $100 \mathrm{U} / \mathrm{ml}$ penicillin were from Beyotime Institute of Biotechnology (Shanghai, China), TRIzol reagent and the reverse transcription kit were from Takara Bio, Inc. (Tokyo, Japan), primers were from Wuhan Qingke Innovative Biotechnology Co., Ltd. (Wuhan, China), NP-40 lysis buffer and the BCA protein assay kit were from Beyotime Institute of Biotechnology. Rabbit anti-P-HSL ser563 and rabbit anti-P-HSL ser660 (cat. nos. 4139 and 4126, respectively) were from Cell Signaling Technology Inc. (Danvers, MA, USA), rabbit anti- $\beta$-actin (cat. no. TDY051) was from Beijing TDY Biotech Co., Ltd. (Beijing, China).

WAT collection and culture. Adult male Sprague-Dawley rats are purchased from the Experimental Animal Center of Wuhan University (Wuhan, China). All animal experiments were performed in compliance with the Principles for Care and Use of Laboratory Animals approved by the Laboratory of Tongji Medical College, Huazhong University of Science and Technology (Wuhan, China). The animals were housed in a room with temperature controlled at $20 \pm 5^{\circ} \mathrm{C}$ and $55 \pm 5 \%$ humidity, under a 12-h light-dark cycle. The rats are fed with standard rat chow (23). Rats with a weight of $300 \pm 30 \mathrm{~g}$ were used during the experiments. The rats were weighed and anesthetized, and the retroperitoneal WAT was rapidly excised in an aseptic laparotomy procedure (34). The explants of WAT were immediately placed in a culture dish containing pre-warmed $\left(37^{\circ} \mathrm{C}\right)$ phosphate-buffered saline (PBS) solution, and washed briefly to dispel blood. The explants were transferred, discarding the tissue residue and fat droplets, into preheated PBS in another culture dish with. A surgical scalpel was used to cut the explants into uniform sections of $\sim 100 \pm 5 \mathrm{mg}$. The sections were gently washed with PBS, resuspended in PBS, and left standing for $5 \mathrm{~min}$ to allow the sections rich in adipocytes to float. The floating sections were collected and transferred into a culture dish containing $25 \mathrm{ml}$ of serum-free M199 with $25 \mathrm{mM}$ HEPES and antibiotics (100 U/ml penicillin-G and $100 \mu \mathrm{g} / \mathrm{ml}$ streptomycin), and cultured at $37^{\circ} \mathrm{C}$ with an atmosphere of $95 \%$ air and $5 \% \mathrm{CO}_{2}$ for $24 \mathrm{~h}$. Studies have shown that live adipocytes can survive in fresh adipose tissue and in adipose tissue cultivated for $24 \mathrm{~h}$, with survival rates of $94 \%$ each and $87 \%$, respectively (35). At the end of the 24-h incubation period, the dish was removed from the incubator, and pre-warmed PBS was used to clean the adipose tissue, using a sterile gauze to dry the tissues. The tissue sections were distributed into a 24-well culture plate (100 mg tissue/well). The wells are then filled with $1 \mathrm{ml}$ Krebs-Ringer buffer containing $25 \mathrm{mM}$ HEPES, $5.5 \mathrm{mM}$ glucose, and $2 \%(\mathrm{w} / \mathrm{v})$ BSA. Immediately, the cultures are treated with normal saline (vehicle), $750 \mathrm{nM}$ ISO [which is known to stimulate the release of NEFA in rat WAT through activation of the $\beta$-adrenergic receptors (36)] as a positive control, or different concentrations of JW extract, JE extract, SW extract, SE extract, RW extract and RW extract. The 

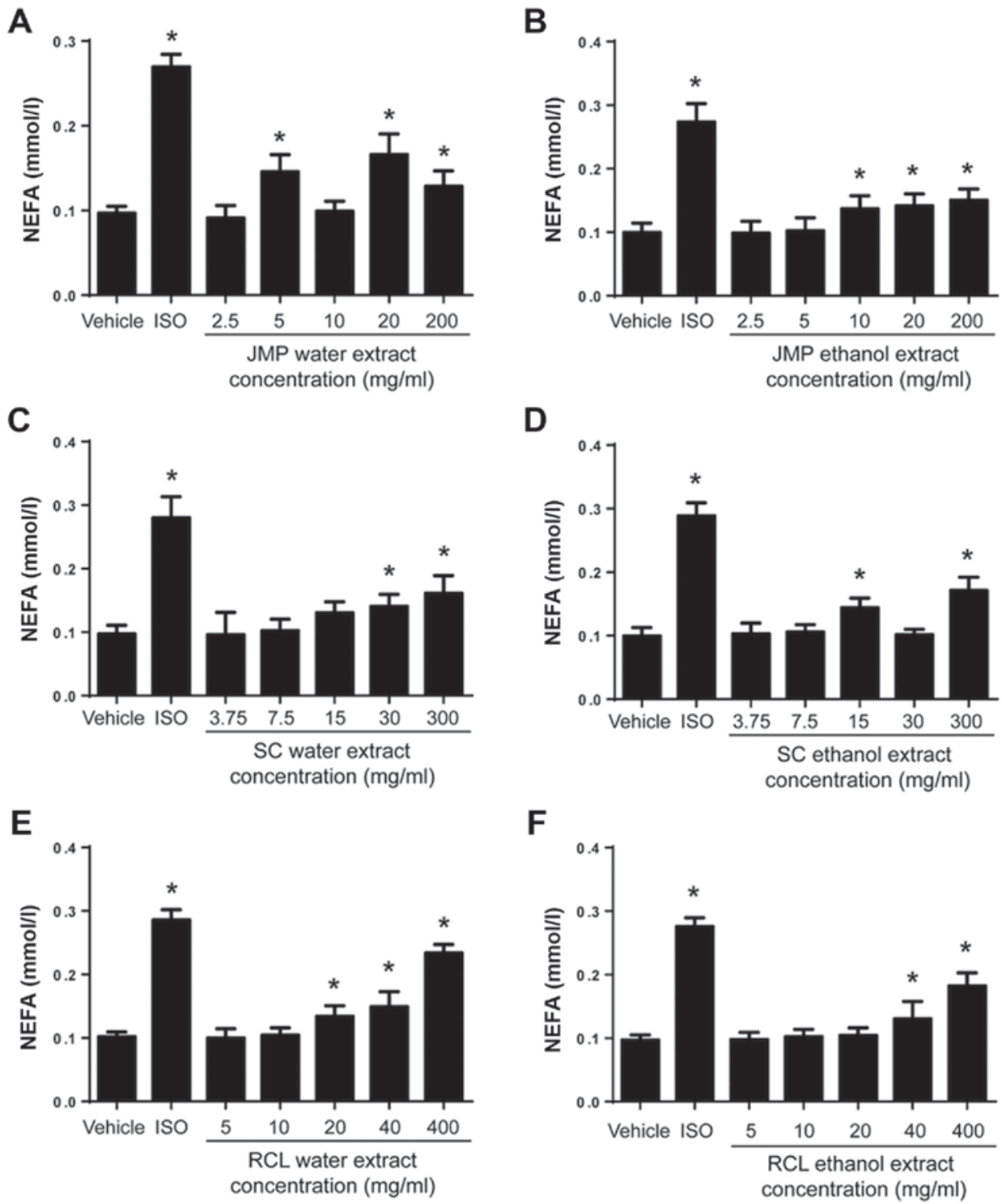

Figure 1. Stimulatory effects of JMP, SC and RCL on the release of NEFA in cultured rat WAT. NEFA release was measured following stimulation with ISO (750 nmol/l) and the indicated concentration of (A) JMP water extract (2.5-200 mg/ml), (B) JMP ethanol extract (2.5-200 mg/ml), (C) SC water extract (3.75-300 mg/ml), (D) SC ethanol extract (3.75-300 mg/ml), (E) RCL water extract (5-400 mg/ml) and (F) CL ethanol extract (5-400 mg/ml) in cultured rat WAT for $90 \mathrm{~min}$. Data are expressed as the mean \pm standard error of the mean of at least three independent experiments, each performed in triplicate ( $\mathrm{n}=3$ ). ${ }^{*} \mathrm{P}<0.05$ vs. vehicle. WAT, white adipose tissue; NEFA, non-esterified fatty acid; vehicle, normal saline; ISO, isoproterenol; JMP, jueming prescription; SC, semen cassia; RCL, Rhizoma Curcumae Longae.

cultures were incubated at $37^{\circ} \mathrm{C}$ in $5 \% \mathrm{CO}_{2}$ and $95 \%$ humidity for $90 \mathrm{~min}$. At the end of the incubation period, the conditioned media were collected from each well. The NEFA and glycerol content were measured using NEFA and glycerol assay kits.

NEFA and glycerol assessment as measures of lipolysis. The assessment of lipolysis was measured by NEFA and glycerol release from WAT into the incubation media. The NEFA and glycerol concentrations in the conditioned media from all samples were determined according to the manufacturer's instructions of the commercial NEFA and glycerol assay kits. The results are expressed as mmol/l of NEFA and mmol/l of glycerol released per $100 \mathrm{mg}$ WAT.

Western blot analysis. The expression of proteins was determined using western blot analysis. The explants of WAT were collected and washed in PBS. The total cellular protein was extracted using NP-40 lysis buffer containing phenylmethanesulphonyl fluoride. The protein concentrations were measured using a BCA protein assay kit according to the manufacturer's instructions. Equal quantities $(40 \mu \mathrm{g})$ of protein samples were separated by 10 or $12 \%$ SDS-PAGE electrophoresis, and then transferred onto polyvinylidene fluoride membranes. The membranes were blocked with $5.0 \%$ fat-free dry milk in Tris-buffered saline and Tween-20 (TBST) at room temperature for $2 \mathrm{~h}$ and incubated with rabbit anti-P-HSL Ser563 $(1: 1,000)$, rabbit anti-P-HSL Ser660 (1:1,000), and rabbit anti- $\beta$-actin $(1: 2,000)$ at $4^{\circ} \mathrm{C}$ overnight. Following washing with TBST four times, the membranes were incubated with the corresponding HRP-conjugated secondary antibodies (cat. no. 074-1506, 1:3,000; KPL, Inc., Gaithersburg, MD, USA) for $1 \mathrm{~h}$ at room temperature on a rocker with gentle agitation. 
A

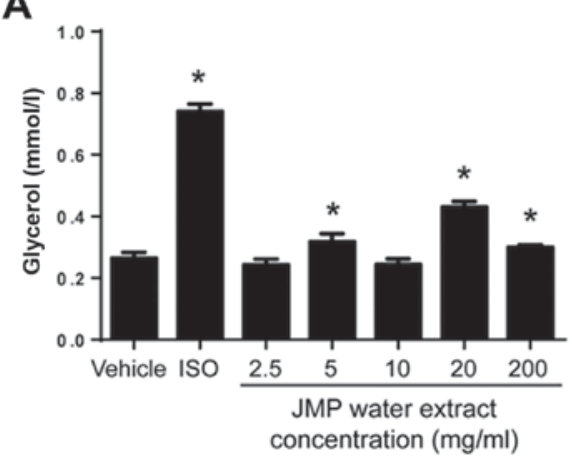

C

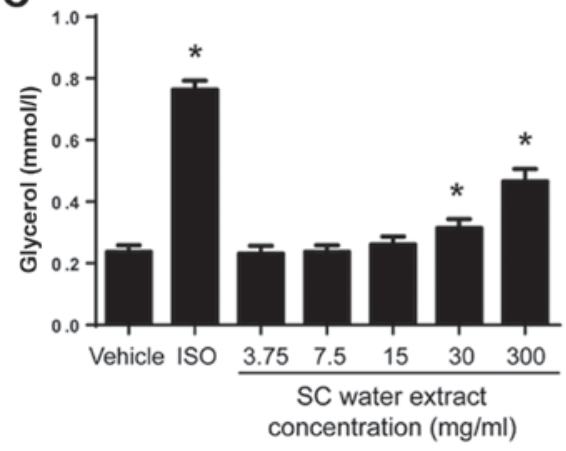

E

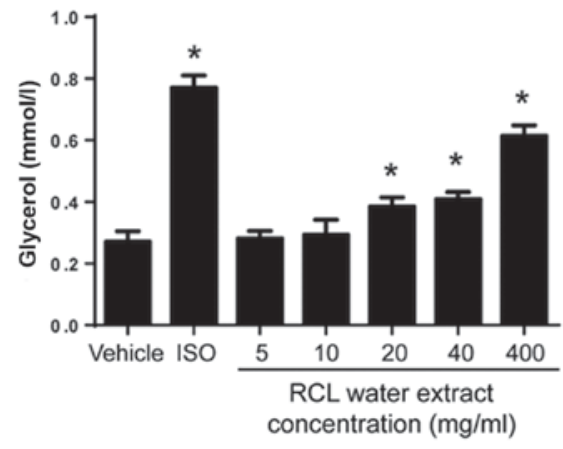

B

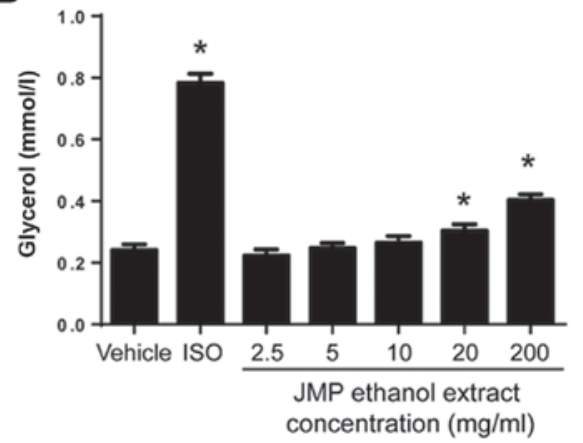

D

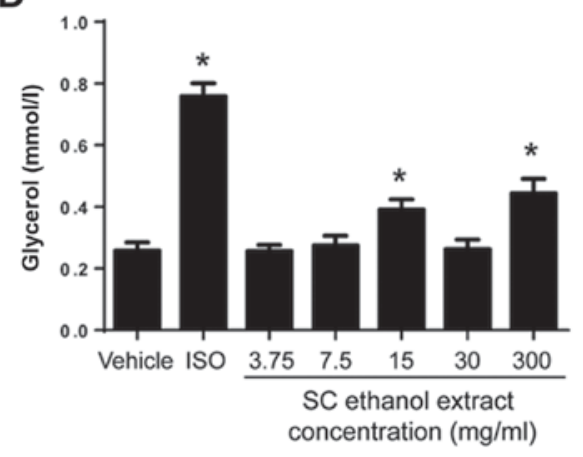

$\mathbf{F}$

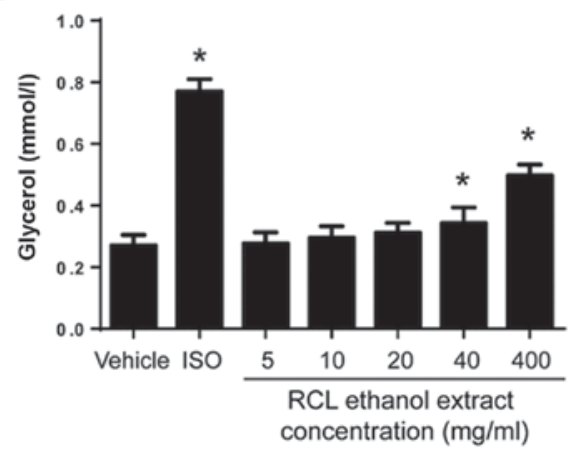

Figure 2. Stimulatory effects of JMP, SC and RCL on glycerol release in cultured rat WAT. Glycerol release was measured following stimulation with ISO $(750 \mathrm{nmol} / \mathrm{l})$ and the indicated concentration of (A) JMP water extract $(2.5-200 \mathrm{mg} / \mathrm{ml})$, (B) JMP ethanol extract $(2.5-200 \mathrm{mg} / \mathrm{ml})$, (C) SC water extract (3.75-300 mg/ml), (D) SC ethanol extract (3.75-300 mg/ml), (E) RCL water extract (5-400 mg/ml) and (F) CL ethanol extract (5-400 mg/ml) in cultured rat WAT for $90 \mathrm{~min}$. Data are expressed as the mean \pm standard error of the mean of at least three independent experiments, each performed in triplicate ( $\mathrm{n}=3$ ). ${ }^{*} \mathrm{P}<0.05$ vs. vehicle. WAT, white adipose tissue; vehicle, normal saline; ISO, isoproterenol; JMP, jueming prescription; SC, semen cassia; RCL, Rhizoma Curcumae Longae.

The bands were visualized using ECL reagent, and the band intensities were quantified by densitometry using Image J software version 2.0 (National Institutes of Health, Bethesda, MD, USA). The results are presented as the ratio of the optical density of the target bands to $\beta$-actin.

Histological analysis. The explants of WAT treated with different concentrations of JCM, SC, RLC were collected, and sections of each explant were fixed in $4 \%$ paraformaldehyde for further embedding in paraffin wax. The tissues blocks were further processed using a routine procedure for hematoxylin and eosin $(\mathrm{H} \& \mathrm{E})$ staining. The stained adipose tissue sections were observed and images were captured under an optical microscope (magnification, $\mathrm{x} 400$ ).

Statistical analysis. All experiment data are presented as the mean \pm standard error of the mean of three or more independent experiments. Statistical significance was determined using GraphPad Prism 6.0 software (GraphPad Software, Inc., La Jolla, CA, USA) by one-way analysis of variance. $\mathrm{P}<0.05$ was considered to indicate a statistically significant difference.

\section{Results}

Stimulatory effects of JMP, SC and RCL on the release of NEFA in cultured rat WAT. To determine whether JMP and its ingredients, SC and RCL, can stimulate lipolysis in rat WAT, the present study investigated the release of NEFA from JMP-, $\mathrm{SC}$ - and RCL-treated rat WAT in vitro. The results revealed that $750 \mathrm{nmol} / 1$ ISO significantly increased the release of NEFA, compared with vehicle (Fig. 1). Furthermore, the rat WAT was treated with JW extract at various concentrations (2.5, 5.0, 10, 20 and $200 \mathrm{mg} / \mathrm{ml}$ ). Compared with the vehicle, the release of NEFA was significantly increased in WAT in the rats treated with JMP concentrations of 5.0, 20 and $200 \mathrm{mg} / \mathrm{ml}$, and the 


\section{Veh ISO JW JE SW SE RW RE}

\section{P-HSL Ser563 _. - - -}

P-HSL Ser660 —_- -
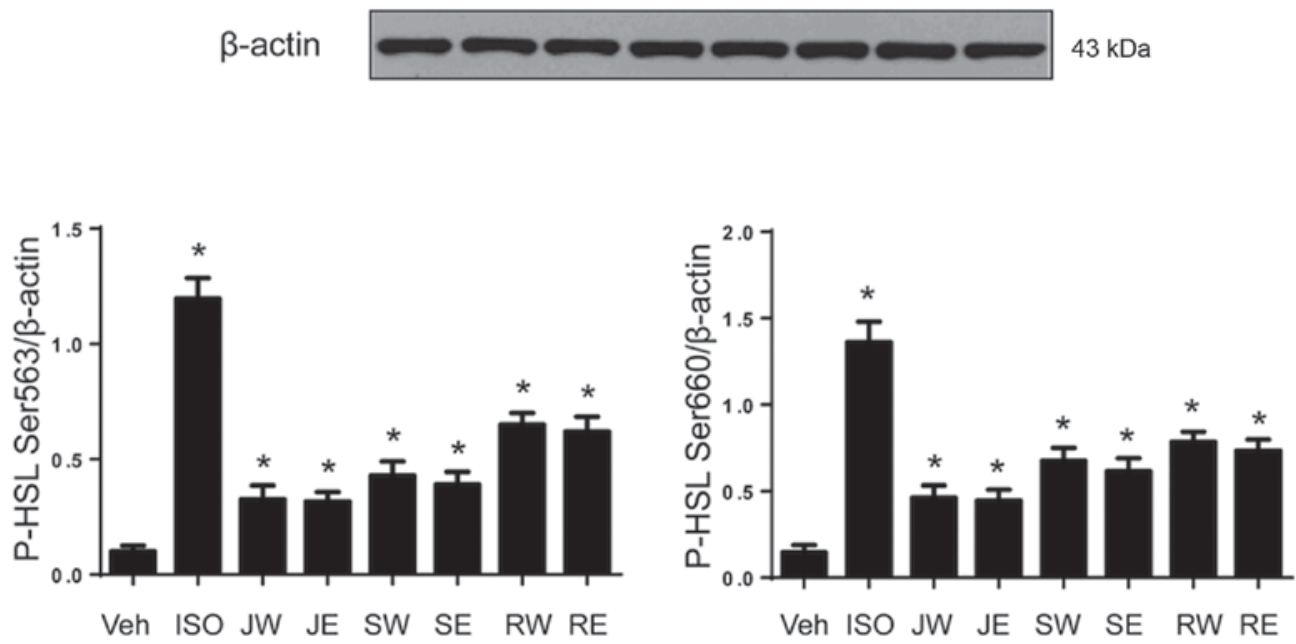

Figure 3. JMP, SC and RCL upregulate p-HSL Ser563 and p-HSL Ser660 in cultured rat WAT. Levels P-HSL Ser563 and P-HSL Ser660 following treatment with JW, JE, SW, SE, RW and RE for $90 \mathrm{~min}$. "P<0.05 vs. Veh. WAT, white adipose tissue; Veh, vehicle, normal saline; ISO, isoproterenol; JMP, jueming prescription; SC, semen cassiae; RCL, Rhizoma Curcumae Longae; P-HSL, phosphorylated of hormone-sensitive lipase; JW, $20 \mathrm{mg} / \mathrm{ml} \mathrm{JMP}$ water extract; JE, $200 \mathrm{mg} / \mathrm{ml} \mathrm{JMP}$ ethanol extract; SW, $300 \mathrm{mg} / \mathrm{ml} \mathrm{SC}$ water extract; SE, $300 \mathrm{mg} / \mathrm{ml} \mathrm{SC}$ ethanol extract; RW, $400 \mathrm{mg} / \mathrm{ml} \mathrm{RCL}$ water extract; RE, $400 \mathrm{mg} / \mathrm{ml} \mathrm{RCL}$ ethanol extract.

maximum effect was observed at a dose of $20 \mathrm{mg} / \mathrm{ml}$ (Fig. 1A). JE extract stimulated the release of NEFA at the concentrations of 10,20 and $200 \mathrm{mg} / \mathrm{ml}$ in a dose-dependent manner, compared with the vehicle-treated group, with the maximum effect at $200 \mathrm{mg} / \mathrm{ml}$ (Fig. 1B). Treatment with 30 and $300 \mathrm{mg} / \mathrm{ml} \mathrm{SW}$ extract stimulated the release of NEFA in a dose-dependent manner, with the maximum effect at $300 \mathrm{mg} / \mathrm{ml}$, compared with the vehicle-treated group (Fig. 1C). Treatment with 15 and $300 \mathrm{mg} / \mathrm{ml} \mathrm{SE}$ extract stimulated the release of NEFA, with the maximum effect at a dose of $300 \mathrm{mg} / \mathrm{ml}$, however this effect was not dose-dependent manner (Fig. 1D). Treatment with 20, 40 and $400 \mathrm{mg} / \mathrm{ml} \mathrm{RW} \mathrm{extract,} \mathrm{and} 40$ and $400 \mathrm{mg} / \mathrm{ml}$ RE extract stimulated the release of NEFA in a dose-dependent manner. The maximum effects of RW extract and RE extract were observed at $400 \mathrm{mg} / \mathrm{ml}$ (Fig. 1E and F).

Stimulatory effects of JMP, SC and RCL on glycerol release in cultured rat WAT. The present study also determined the release of glycerol from the rat WAT treated with ISO, JMP and its ingredients, SC and RCL. Consistent with the release of NEFA, the effects on lipolysis by the treatments on glycerol were similar in the medium from the cultured rat WAT. Treatment with $750 \mathrm{nmol} / 1 \mathrm{ISO}, 5.0,20$ and $200 \mathrm{mg} / \mathrm{ml}$ JW extract (maximum at $20 \mathrm{mg} / \mathrm{ml}$ ), 20 and $200 \mathrm{mg} / \mathrm{ml} \mathrm{JE}$ extract (dose-dependent with maximum at $200 \mathrm{mg} / \mathrm{ml}$ ), 30 and $300 \mathrm{mg} / \mathrm{ml} \mathrm{SW}$ extract (dose-dependent with the maximum at $300 \mathrm{mg} / \mathrm{ml}), 15$ and $300 \mathrm{mg} / \mathrm{ml} \mathrm{SE} \mathrm{extract}$ (maximum at $300 \mathrm{mg} / \mathrm{ml}$ ), 20, 40 and $400 \mathrm{mg} / \mathrm{ml} \mathrm{RW}$ extract (dose-dependent, maximum at $400 \mathrm{mg} / \mathrm{ml}$ ), 40 and $400 \mathrm{mg} / \mathrm{ml}$ RE extract (dose-dependent, maximum at $400 \mathrm{mg} / \mathrm{ml}$ ) stimulated the release of glycerol, compared with release in the vehicle-treated group (Fig. 2A-F).
JMP, SC and RCL upregulate the phosphorylation of HSL at Ser563 and Ser660 in cultured rat WAT. The HSL at Ser563 and Ser660 are major phosphorylation sites associated with HSL activity, and are well known markers of lipolysis $(14,18-22)$. ISO is known to phosphorylate HSL at Ser563 and Ser660 sites, and results in increasing lipolysis, which is used as a positive control $(21,22)$. According to the results of the release of NEFA and glycerol (Figs. 1 and 2), the present study examined the phosphorylation levels of HSL at Ser563 and Ser660 in cultured rat WAT treated with $20 \mathrm{mg} / \mathrm{ml}$ JW extract, $200 \mathrm{mg} / \mathrm{ml} \mathrm{JE}$ extract, $300 \mathrm{mg} / \mathrm{ml} \mathrm{SW}$ extract, $300 \mathrm{mg} / \mathrm{ml} \mathrm{SE}$ extract, $400 \mathrm{mg} / \mathrm{ml} \mathrm{RW}$ extract and $400 \mathrm{mg} / \mathrm{ml}$ RE extract. As shown in Fig. 3, consistent with the release of NEFA and glycerol, $750 \mathrm{nmol} / 1$ ISO, JW extract, JE extract, SW extract, SE extract, RW extract and RE extract significantly upregulated the phosphorylation levels of HSL at Ser563 (P-HSL Ser563) and Ser660 (P-HSL Ser660), compared with that in the vehicle-treated group. This suggested that JMP, SC and RLC significantly upregulated the expression of P-HSL Ser563 and P-HSL Ser660 in the stimulation of lipolysis in rat WAT in vitro. RCL had the most marked effect on increasing the levels of P-HSL Ser563 and P-HSL Ser660, particularly RW extract (Fig. 3).

$J M P, S C$ and RCL decrease the size of adipocytes in cultured rat WAT. To confirm whether JMP, SC and RCL reduce the size of adipocytes, the cultured rat WAT was stained using HE staining. Histological analysis of the adipose tissue to confirmed the sizes of the adipocytes (Fig. 4). The size of adipocytes was markedly smaller in the ISO $(750 \mathrm{~nm})$ group, compared with that in the vehicle group. The adipocytes were also smaller in the $20 \mathrm{mg} / \mathrm{ml} \mathrm{JW}$ extract, $200 \mathrm{mg} / \mathrm{ml} \mathrm{JE}$ extract, 

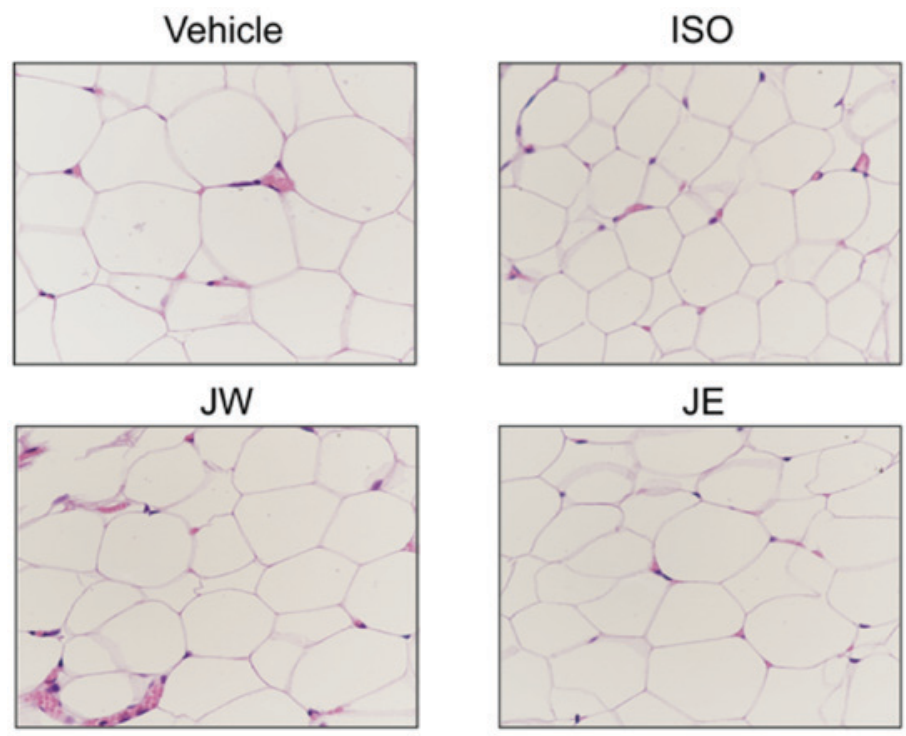

SW

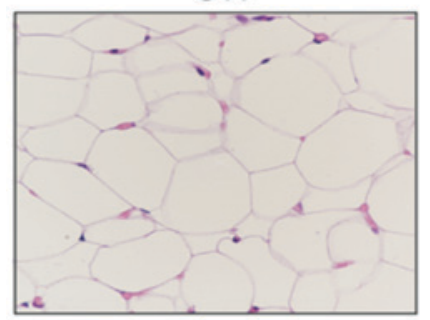

SE

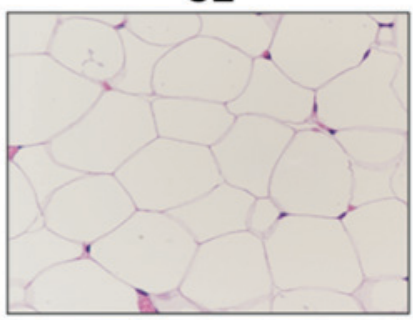

RW

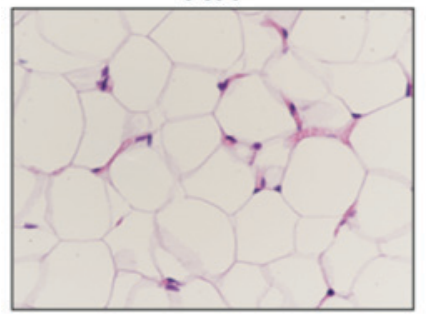

RE

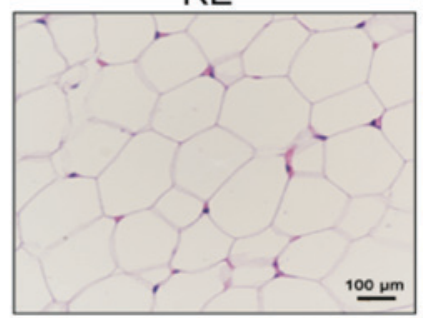

Figure 4. JMP, SC and RCL decrease the size of adipocytes in cultured rat WAT. The cultured rat WAT was stained using hematoxylin and eosin, and observed under a microscope (magnification, x400). The size of adipocytes is shown following treatment with JW, JE, SW, SE, RW and RE for 90 min. WAT, white adipose tissue; vehicle, normal saline; ISO, isoproterenol; JMP, jueming prescription; SC, semen cassiae; RCL, Rhizoma Curcumae Longae; JW, 20 mg/ml JMP water extract; JE, 200 mg/ml JMP ethanol extract; SW, 300 mg/ml SC water extract; SE, 300 mg/ml SC ethanol extract; RW, 400 mg/ml RCL water extract; RE, $400 \mathrm{mg} / \mathrm{ml} \mathrm{RCL}$ ethanol extract.

$300 \mathrm{mg} / \mathrm{ml} \mathrm{SW}$ extract, $300 \mathrm{mg} / \mathrm{ml} \mathrm{SE}$ extract, $400 \mathrm{mg} / \mathrm{ml} \mathrm{RW}$ extract and $400 \mathrm{mg} / \mathrm{ml} \mathrm{RE}$ extract groups, compared with size in the vehicle group.

\section{Discussion}

Obesity is characterized by excess TG accumulation in adipocytes of WAT (1). Lipolysis is the process of TG breakdown in WAT, resulting in the release of NEFA and glycerol (2-5,37). Experiments using cultured adipocytes, WAT explants and obese animals have demonstrated that increased phosphorylation levels of HSL at Ser563 and Ser660 promote lipolysis (20-22). Chinese herbal medicine is important in the treatment of obesity and enhancement of lipolysis (2,6,23-26). Our previous studies showed that JMP exhibited anti-obesity effects, and enhanced lipolysis in obese rats and patients $(23,27,28)$. SC and RCL extracts also increased lipolysis to result in the release of NEFA and glycerol, and reduce the size of adipocytes $(2,3,6,32)$. In the present study, it was found that JMP and its ingredients, SC and RCL, stimulated lipolysis and upregulated the phosphorylation levels of HSL at Ser563 and Ser660 in cultured rat WAT.

The induction of NEFA and glycerol release in intact WAT explants is used in the assessment of lipolysis (4,36,38-40). ISO, which is known to stimulate the release of NEFA and glycerol in rat WAT through the activation of $\beta$-adrenergic receptors, is used as a positive control (34-36). Therefore, the present study examined the release of NEFA and glycerol from ISO-, JMP-, SC- and RCL-treated rat WAT explants in vitro. ISO significantly increased the release of NEFA and glycerol. The JW, JE, SW, SE, RW and RE extracts also stimulated the release of NEFA and glycerol.

Previous studies have confirmed that the phosphorylation of HSL at Ser563 and Ser660 activates HSL, and increased HSL 
translocates to lipid droplets and enhances lipolysis $(19,20)$. In the present study, ISO, known to phosphorylate HSL at the Ser563 and Ser660 sites and increase lipolysis, was used as a positive control $(21,22)$. It was found that the ISO, JW, JE, SW, SE, RW and RE extracts significantly upregulated the phosphorylation levels of HSL at Ser563 and Ser660 in the cultured rat WAT. The effects of the single herb SC and RCL extracts were superior to that of JMP. It was hypothesized that SC and RCL in JMP may induce a chemical reaction or the formation of certain compounds, leading JMP to have multiple effects and weakened lipolysis. This requires further investigation in the future. The RCL single herb had the most marked effect on increasing the levels of P-HSL Ser563 and P-HSL Ser660, particularly RW extract. This is consistent with the effects of water extracts of herbs used clinically for treating obese patients $(27,41)$.

The present study also showed that ISO and the JW, JE, SW, SE, RW and RE extracts reduced the size of adipocytes in the cultured rat WAT. Histological analyses of the WAT confirmed that the sizes of the adipocytes were smaller on visualization under a microscope (magnification, $\mathrm{x} 400$ ) in the ISO-, JMP-, SCand RCL-treated WAT, compared with the vehicle-treated group.

In conclusion, the results of the present study indicated that the effects of JMP and its ingredients, SC and RCL, on the stimulation of lipolysis were responsible for increases in the release of NEFA and glycerol, and a reduction in the size of adipocytes in an ex vivo organ culture method. This may be through upregulating the phosphorylation of HSL at Ser563 and Ser660 in rat WAT.

\section{Acknowledgements}

This study was supported by the National Natural Science Foundation of China (grant no. 81673657), the Ministry of Education Specialized Research Foundation for the Doctoral Program of Education (grant no. 20110142110016) and the Innovation Foundation of Huazhong University of Science and Technology Ph.D. Dissertation (grant no. 0118550034).

\section{References}

1. Frühbeck G, Gómez-Ambrosi J, Muruzábal FJ and Burrell MA: The Adipocyte: A model for integration of endocrine and metabolic signaling in energy metabolism regulation. Am J Physiol Endocrinol Metab 280: E827-E847, 2001.

2. Kim JH, Kim OK, Yoon HG, Park J, You Y, Kim K, Lee YH, Choi KC, Lee J and Jun W: Anti-obesity effect of extract from fermented Curcuma longa L. through regulation of adipogenesis and lipolysis pathway in high-fat diet-induced obese rats. Food Nutr Res 60: 30428, 2016.

3. Lee J, Yoon HG, Lee YH, Park J, You Y, Kim K, Jang JY, Yang JW and Jun W: The potential effects of ethyl acetate fraction from Curcuma longa L. on lipolysis in differentiated 3T3-L1 adipocytes. J Med Food 13: 364-370, 2010.

4. Stelmanska E, Szrok S and Swierczynski J: Progesterone-induced down-regulation of hormone sensitive lipase (Lipe) and up-regulation of G0/G1 Switch 2 (G0s2) genes expression in inguinal adipose tissue of female rats is reflected bydiminished rate of lipolysis. J Steroid Biochem Mol Biol 147: 31-39, 2015.

5. Duncan RE, Ahmadian M, Jaworski K, Sarkadi-Nagy E and Sul HS: Regulation of lipolysis in adipocytes. Annu Rev Nutr 27: 79-101, 2007

6. Ho JN, Jang JY, Yoon HG, Kim Y, Kim S, Jun W and Lee J: Anti-obesity effect of a standardised ethanol extract from Curcuma longa $\mathrm{L}$. fermented with aspergillus oryzae in ob/ob mice and primary mouse adipocytes. J Sci Food Agric 92: 1833-1840, 2012 .
7. Baillie GS: Compartmentalized signalling: Spatial regulation of cAMP by the action of compartmentalized phosphodiesterases. FEBS J 276: 1790-1799, 2009.

8. Zimmermann R, Strauss JG, Haemmerle G, Schoiswohl G, Birner-Gruenberger R, Riederer M, Lass A, Neuberger G, Eisenhaber F, Hermetter A and Zechner R: Fat mobilization in adipose tissue is promoted by adipose triglyceride lipase. Science 306: 1383-1386, 2004.

9. Carmen GY and Víctor SM: Signalling mechanisms regulating lipolysis. Cell Signal 18: 401-408, 2006

10. Frayn KN, Karpe F, Fielding BA, Macdonald IA and Coppack SW: Integrative physiology of human adipose tissue. Int J Obes Relat Metab Disord 27: 875-888, 2003.

11. Egan JJ, Greenberg AS, Chang MK, Wek SA, Moos MC Jr and Londos C: Mechanism of hormone-stimulated lipolysis in adipocytes: Translocation of hormone-sensitive lipase to the lipid storage droplet. Proc Natl Acad Sci USA 89: 8537-8541, 1992.

12. Kul'berg AY, Shmeleva NE and Bartova LM: The homoreagent system and immunobiological properties of the products of proteolytic hydrolysis of the antigen-antibody complex. Bull Exp Biol Med 73: 75-77, 1972.

13. Shen WJ, Patel S, Miyoshi H, Greenberg AS and Kraemer FB: Functional interaction of hormone-sensitive lipase and perilipin in lipolysis. J Lipid Res 50: 2306-2313, 2009.

14. Anthonsen MW, Rönnstrand L, Wernstedt C, Degerman E and Holm C: Identification of novel phosphorylation sites in hormone-sensitive lipase that are phosphorylated in response to isoproterenol and govern activation properties in vitro. J Biol Chem 273: 215-221, 1998.

15. Greenberg AS, Shen WJ, Muliro K, Patel S, Souza SC, Roth RA and Kraemer FB: Stimulation of lipolysis and hormone-sensitive lipase via the extracellular signal-regulated kinase pathway. J Biol Chem 276: 45456-45461, 2001.

16. Subramanian V, Rothenberg A, Gomez C, Cohen AW, Garcia A, Bhattacharyya S, Shapiro L, Dolios G, Wang R, Lisanti MP and Brasaemle DL: Perilipin a mediates the reversible binding of CGI-58 to lipid droplets in 3T3-L1 adipocytes. J Biol Chem 279: 42062-42071, 2004.

17. Frühbeck G, Méndez-Giménez L, Fernández-Formoso JA, Fernández S and Rodríguez A: Regulation of adipocyte lipolysis. Nutr Res Rev 27: 63-93, 2014.

18. Roepstorff C, Vistisen B, Donsmark M, Nielsen JN, Galbo H, Green KA, Hardie DG, Wojtaszewski JF, Richter EA and Kiens B: Regulation of hormone-sensitive lipase activity and Ser563 and Ser565 phosphorylation in human skeletal muscle during exercise. J Physiol 560: 551-562, 2004.

19. Watt MJ, Holmes AG, Pinnamaneni SK, Garnham AP, Steinberg GR, Kemp BE and Febbraio MA: Regulation of HSL serine phosphorylation in skeletal muscle and adipose tissue. Am J Physiol Endocrinol Metab 290: E500-E508, 2006.

20. Kim SJ, Tang T, Abbott M, Viscarra JA, Wang Y and Sul HS: AMPK phosphorylates desnutrin/ATGL and hormone-sensitive lipase to regulate lipolysis and fatty acid oxidation within adipose tissue. Mol Cell Biol 36: 1961-1976, 2016.

21. Ding L, Zhang F, Zhao MX, Ren XS, Chen Q, Li YH, Kang YM and Zhu GQ: Reduced lipolysis response to adipose afferent reflex involved in impaired activation of adrenoceptor-cAMP-PKA-hormone sensitive lipase pathway in obesity. Sci Rep 6: 34374, 2016.

22. Larsson S, Jones HA, Göransson O, Degerman E and Holm C: Parathyroid hormone induces adipocyte lipolysis via PKA-mediated phosphorylation of hormone-sensitive lipase. Cell Signal 28: 204-213, 2016.

23. Yang L, Lu K, Wen XY, Liu H, Chen AP, Xu MW, Zhang H and Yu J: Jueming prescription reduces body weight by increasing the mRNA expressions of beta3-adrenergic receptor and uncoupling protein-2 in adipose tissue of diet-induced obese rats. Chin J Integr Med 18: 775-781, 2012.

24. He GW, Qu WJ, Fan B, Jing R and He R: The protective effect of Yi-Qi-Yang-Yin-Ye, a compound of traditional chinese herbal medicine in diet-induced obese rats. Am J Chin Med 36: 705-717, 2008.

25. He CY, Wang WJ, Li B, Xu DS, Chen WH, Ying J and He YM: Clinical research of Yiqi Sanju formula in treating central obese men at high risk of metabolic syndrome. Zhong Xi Yi Jie He Xue Bao 5: 263-267, 2007 (In Chinese).

26. Jung DW, Lee OH and Kang IJ: Sanguisorba officinalis L. extracts exert antiobesity effects in 3T3-L1 adipocytes and C57BL/6J mice fed high-fat diets. J Med Food 19: 768-779, 2016. 
27. Wen XY, Liu H, Zhang CY and Pan QY: Study on the clinical efficacy of weight loss and the effect on lipid metabolism of Jueming prescription. Chin Hosp Pharm J 30: 2097-2100, 2010 (In Chinese)

28. Wen XY, Hu QJ, Liu H, Xu MW, Xiong L and Chen AP: Effect of Jueming prescription on the fatty liver and its SREBP-1c expression in the obese rats. Chin J Hosp Pharm 29: 1518-1521, 2009 (In Chinese).

29. Zhang L: Experience of 30 cases of obesity treated by traditional chinese medicine. Chin J Mod Drug Appl 9: 244-245, 2015 (In Chinese).

30. Li J, Chen XY, Zhao LJ: Traditional chinese medicine syndrome differentiation of obesity disease. Chin J Trad Chin Med Pharm 18: 59-60, 2003 (In Chinese).

31. He JY,Liu SQ, Peng YF and Chen ZL: Study of the mechanism of Cassia obtusifolia L. in decreasing blood-lipid. Chin Pharm 14 202-204, 2003 (In Chinese).

32. Xie W, Zhao Y and Du L: Emerging approaches of traditional chinese medicine formulas for the treatment of hyperlipidemia. J Ethnopharmacol 140: 345-367, 2012.

33. Wen X, Zeng Y, Liu L, Zhang H, Xu W, Li N and Jia X: Zhenqing recipe alleviates diabetic nephropathy in experimental type 2 diabetic rats through suppression of SREBP-1c. J Ethnopharmacol 142: 144-150, 2012.

34. Ghorbani A, Omrani GR, Hadjzadeh MA and Varedi M: Proinsulin C-peptide inhibits lipolysis in diabetic rat adipose tissue through phosphodiestrase-3B enzyme. Horm Metab Res 45: 221-225, 2013.
35. Ghorbani A, Omrani GR, Hadjzadeh MA and Varedi M: Effects of rat C-peptide-II on lipolysis and glucose consumption in cultured rat adipose tissue. Exp Clin Endocrinol Diabetes 119: 343-347, 2011.

36. Møller CL, Pedersen SB, Richelsen B, Conde-Frieboes KW, Raun K, Grove KL and Wulff BS: Melanocortin agonists stimulate lipolysis in human adipose tissue explants but not in adipocytes. BMC Res Notes 8: 559, 2015.

37. Gaidhu MP, Anthony NM, Patel P, Hawke TJ and Ceddia RB: Dysregulation of lipolysis and lipid metabolism in visceral and subcutaneous adipocytes by high-fat diet: Role of ATGL, HSL and AMPK. Am J Physiol Cell Physiol 298: C961-C971, 2010.

38. Chen N, Zhou L, Zhang Z, Xu J, Wan Z and Qin L: Resistin induces lipolysis and suppresses adiponectin secretion in cultured human visceral adipose tissue. Regul Pept 194: 49-54, 2014.

39. Karbowska J and Kochan Z: Fat-reducing effects of dehydroepiandrosterone involve upregulation of ATGL and HSL expression and stimulation of lipolysis in adipose tissue. Steroids 77: 1359-1365, 2012.

40. Niang F, Benelli C, Ribière C, Collinet M, Mehebik-Mojaat N, Penot G, Forest C and Jaubert AM: Leptin induces nitric oxide-mediated inhibition of lipolysis and glyceroneogenesis in rat white adipose tissue. J Nutr 141: 4-9, 2011.

41. Tang HZ: Clinical observation on 60 cases of simple obesity treated by Jianfei prescription. Hunan Trand Chin Med 23: 17-18, 2007 (In Chinese) 